\title{
Automated versus non-automated weaning for reducing the duration of mechanical ventilation for critically ill adults and children: a cochrane systematic review and meta-analysis
}

Louise Rose $e^{1,2,3,4,5^{*}}$, Marcus J Schultz ${ }^{6}$, Chris R Cardwell7 , Philippe Jouvet $^{8}$, Danny F McAuley ${ }^{9}$ and Bronagh Blackwood ${ }^{10}$

\begin{abstract}
Introduction: Automated weaning systems may improve adaptation of mechanical support for a patient's ventilatory needs and facilitate systematic and early recognition of their ability to breathe spontaneously and the potential for discontinuation of ventilation. Our objective was to compare mechanical ventilator weaning duration for critically ill adults and children when managed with automated systems versus non-automated strategies. Secondary objectives were to determine differences in duration of ventilation, intensive care unit (ICU) and hospital length of stay (LOS), mortality, and adverse events.

Methods: Electronic databases were searched to 30 September 2013 without language restrictions. We also searched conference proceedings; trial registration websites; and article reference lists. Two authors independently extracted data and assessed risk of bias. We combined data using random-effects modelling.

Results: We identified 21 eligible trials totalling 1,676 participants. Pooled data from 16 trials indicated that automated systems reduced the geometric mean weaning duration by 30\% (95\% confidence interval (Cl) 13\% to $45 \%)$, with substantial heterogeneity $\left(1^{2}=87 \%, P<0.00001\right)$. Reduced weaning duration was found with mixed or medical ICU populations (42\%, 95\% Cl 10\% to 63\%) and Smartcare/PS ${ }^{\text {TM }}$ (28\%, 95\% Cl 7\% to 49\%) but not with surgical populations or using other systems. Automated systems reduced ventilation duration with no heterogeneity (10\%, 95\% CI 3\% to 16\%) and ICU LOS (8\%, 95\% CI 0\% to 15\%). There was no strong evidence of effect on mortality, hospital LOS, reintubation, self-extubation and non-invasive ventilation following extubation. Automated systems reduced prolonged mechanical ventilation and tracheostomy. Overall quality of evidence was high.
\end{abstract}

Conclusions: Automated systems may reduce weaning and ventilation duration and ICU stay. Due to substantial trial heterogeneity an adequately powered, high quality, multi-centre randomized controlled trial is needed.

\section{Introduction}

Serious physiological and psychological sequelae are associated with protracted invasive mechanical ventilation, necessitating efficient processes to safely reduce and remove ventilator support, termed weaning [1,2]. Tools such as weaning protocols and automated systems may facilitate systematic and early recognition of spontaneous

\footnotetext{
*Correspondence: louise.rose@utoronto.ca

'Department of Critical Care Medicine, Sunnybrook Health Sciences Centre, 2075 Bayview Ave, Toronto, ON M4N 3M5, Canada

${ }^{2}$ Lawrence S. Bloomberg Faculty of Nursing, University of Toronto, 155

College St, Toronto, Ontario M5T IP8, Canada

Full list of author information is available at the end of the article
}

breathing ability and the potential for ventilation discontinuation. These tools may reduce practice variation and improve efficiency by emphasizing timely and objective decision making [3]. A 2010 Cochrane review evaluating the effectiveness of protocolized versus non-protocolized weaning [4] found evidence of reduced duration of mechanical ventilation, weaning and intensive care unit (ICU) stay using standardized weaning protocols but also significant heterogeneity among studies.

Weaning from mechanical ventilation traditionally occurs via clinician-directed adjustments to the level of assistance provided by the ventilator culminating in 
a spontaneous breathing trial. Through continuous monitoring and real-time interventions, automated systems theoretically provide improved adaptation of ventilatory support to patients' needs when compared to cliniciandirected weaning [5]. Several such systems are now commercially available and include Smartcare/PS ${ }^{\mathrm{mu}}$ (DrägerMedical, Lübeck, Germany), Adaptive Support Ventilation (ASV) (HamiltonMedical, Bonaduz, Switzerland), Automode (Siemens, Solna, Sweden), Proportional Assist Ventilation (PAV+ The University of Manitoba, Canada used under license by Covidien, Minneapolis, US), Mandatory Minute Ventilation (MMV) (Dräger Medical), Proportional Pressure Support (PPS) (DrägerMedical), Neurally Adjusted Ventilatory Assist (NAVA) (Maquet, Solna, Sweden) and Intellivent- $\mathrm{ASV}^{\circ}$ (Hamilton Medical, Rhäzüns, Switzerland). (see Additional file 1). Automation of weaning potentially reduces avoidable delays as it is less reliant on clinician recognition of changes in the patient's weaning status which can be influenced by clinician availability, expertise and work load.

There is a pressing imperative to identify efficiencies in weaning resulting in reduced ventilation duration to prevent ventilator associated morbidity and mortality, and also to offer solutions to existing constraints in critical care services. The number of patients receiving mechanical ventilation continues to increase due to improved patient survival and population aging [6]. Costs of care provision to these patients are substantial [7]. This increased demand is occurring alongside a reduced supply of healthcare professionals qualified and skilled in mechanical ventilation management and its weaning $[8,9]$. If efficacious in terms of clinical outcomes, automated weaning systems could enable safe and efficient management of weaning despite predicted staffing shortages. Therefore, our primary objective was to compare the total weaning duration, defined as study randomization to successful extubation for critically ill ventilated adults and children managed with an automated weaning system versus non-automated strategies. Secondary objectives were to determine differences in ventilation duration, ICU and hospital lengths of stay (LOS), mortality and adverse events related to early or delayed extubation. More details regarding this review can be found in the Cochrane Database of Systematic Reviews [10].

\section{Methods}

Trials were eligible if they were randomized, compared automated systems designed to reduce ventilator support based on continuous monitoring of patient tolerance and interpretation of real-time physiological changes to non-automated weaning strategies including standard or usual care and protocolized weaning, and were conducted in critically ill adults and children over four weeks of age. We included adults and children as the same tenets of weaning apply [11]. Research Ethics Board approval and participant consent were not required as this is a systematic review including published and non-published data from existing trials.

Two authors independently searched MEDLINE, EMBASE, Cochrane Central Register of Controlled Trials, CINAHL, LILACS, Web of Science, the Database of Abstracts of Reviews of Effects and the Health Technology Assessment Database up to 30 September 2013. We combined search terms for automated systems with the Cochrane highly sensitive search strategy for randomized controlled trials; language restrictions did not apply. We searched conference proceedings, trial registration websites, reference lists of retrieved studies and review papers and contacted trial authors and content experts.

\section{Data extraction}

Using a standardized form, two authors independently extracted: study design and setting, participant characteristics, inclusion/exclusion criteria, weaning methods (intervention and control arms), sedation strategies and study outcomes. We recorded randomization methods, allocation concealment, blinding, frequency and handling of missing data, adherence to intention-to-treat analysis, and selective outcome reporting. We contacted corresponding authors to seek further clarification on issues of reporting or to obtain additional outcome data. Data extractors were not blinded to study citations.

\section{Assessment of methodological quality}

Two authors independently assessed methodological quality and risk of bias using the domain-based evaluation recommended by The Cochrane Collaboration [12]. Domains judged as high, low or unclear risk of bias included: random sequence generation, allocation concealment, blinding, incomplete outcome data, selective reporting and other biases. We used assessment of risk of bias to perform sensitivity analyses based on methodological quality.

\section{Statistical analyses}

We calculated the difference in means, 95\% confidence intervals (CIs) and the standard error of that difference for continuous outcomes. For dichotomous data, we described treatment effects using risk ratios (RR) and 95\% CIs. We used random-effects models to calculate pooled estimates as heterogeneity was anticipated [13]. As we detected considerable skew in continuous outcomes, we $\log$ transformed data for the primary analysis. For six trials [14-19], we log transformed raw data obtained from corresponding authors. For nine trials [20-28], we used the mean and standard deviation (SD) on the unlogged scale to calculate a mean and SD on the log transformed scale using methods described by Higgins [12]. For six 
trials [29-34], we used the median and interquartile range (IQR) of the unlogged data to approximate the mean if not available using methods described by Hozo and colleagues [35] and calculated an approximate SD on the log scale from the IQR on the log scale [12]. We exponentiated the difference in the mean of a variable on the log scale to provide the ratio of geometric means on the unlogged scale and reported this as a percentage change and 95\% CIs (reduction or increase) in geometric mean for ease of understanding [36]. All analyses were performed in Review Manager 5.2 [37].

\section{Subgroup and sensitivity analyses}

Pre-specified subgroup analyses were performed based on ICU population grouped as either 'medical and mixed' or 'surgical'; the automated system evaluated; and the non-automated weaning strategy used (protocolized versus non-protocolized weaning). Due to limited numbers of studies we collapsed studies of medical or mixed ICU population into one group. We were unable to perform a priori planned subgroup analyses according to weaning classification (simple, difficult, prolonged [2]) as no trials used this taxonomy; adult versus pediatric populations as only one trial recruited children; and sedation strategy as this was inadequately reported in most trials. We conducted sensitivity analyses on unlogged data for all continuous outcomes and on logged data for the primary outcome excluding trials with high risk of bias.

\section{Assessment of heterogeneity and reporting bias}

We evaluated clinical heterogeneity by qualitative assessment of study differences in terms of study population, ICU type, clinician involvement in weaning decision making, and implementation of weaning and extubation processes. Statistical heterogeneity was informally evaluated from forest plots, and more formally using the Chi square test $\left(P<0.05\right.$, significant heterogeneity) and $\mathrm{I}^{2}$ statistic $\left(\mathrm{I}^{2}>50 \%\right.$, moderate to substantial heterogeneity) [12]. We constructed a funnel plot of the treatment effect for the primary outcome against trial precision (standard error). We identified sufficient studies $(\geq 10)$ to formally test for asymmetry [38].

\section{Results}

Of the retrieved 1,249 citations, 21 trials of 1,676 participants were included (Figure 1). Twelve trials recruited mixed or medical ICU populations $(n=871,52 \%)$ [14-16,18,21,23,25,27-31] and nine surgical ICU patients $(\mathrm{n}=805,48 \%) \quad[17,19,20,22,24,26,32-34]$. Eight trials evaluated Smartcare/PS ${ }^{\mathrm{Tw}}$ (DrägerMedical) $(\mathrm{n}=803,48 \%)$ $[14,16,17,23,27,30,31,33]$, seven trials Adaptive Support Ventilation (ASV) (HamiltonMedical) $(\mathrm{n}=424,25 \%)$ $[15,19,20,29,32,34]$, three trials Automode (Siemens) $[22,24,28](\mathrm{n}=78.5 \%)$, one trial Mandatory Minute
Ventilation DrägerMedical) [21], one trial Mandatory Rate Ventilation [26], one trial Proportional Assist Ventilation $\left(\mathrm{PAV}^{+}\right)[18]$, and one trial describing a non-commercial automated system [25]. Sixteen trials $[15,17-24,26,28-32,34]$ used protocolized weaning as the comparator; five $[10,16,25,27,33]$ used usual care (Table 1). Most studies were of high methodological quality (Figure 2) although no studies blinded participants or clinical personnel.

\section{Weaning duration}

Pooled data from 16 trials reporting weaning duration indicated a reduction using automated systems (mean $\log$ hours $-0.36,95 \% \mathrm{CI}-0.59$ to $-0.14, P=0.001$ ), equivalent to a $30 \%$ ( $95 \%$ CI $13 \%$ to $45 \%)$ reduction in the geometric mean. Statistically significant $(P<0.00001)$ and substantial $\left(I^{2}=87 \%\right)$ heterogeneity was noted (Figure 3 ). Subgroup analyses according to ICU population demonstrated reduced weaning duration in trials of mixed/medical ICU patients (mean log hours $-0.55,-1.00$ to -0.10 , $P=0.02 ; 42 \%$ (10\% to $63 \%)$ reduction in geometric mean). No evidence of effect was found in trials including only surgical ICU patients. Smartcare/PS ${ }^{\mathrm{mm}}$ reduced weaning duration (mean $\log$ hours $-0.33,-0.58$ to $-0.09, P=0.008$; $28 \%$ (7\% to $49 \%)$ reduction in geometric mean) whereas ASV (mean log hours $-0.03,-0.11$ to $0.05, P=0.50 ; 3 \%$ (5\% increase to $10 \%$ reduction) reduction in geometric mean) and other systems (mean log hours $-0.54,-1.17$ to $0.08, P=0.09 ; 42 \%$ ( $8 \%$ increase to $69 \%$ reduction) reduction in geometric mean) did not (Figure 4). There was no subgroup difference according to the weaning method used in the control arm with broadly overlapping CIs.

\section{Randomization to first extubation}

Pooled data from eleven trials reporting duration of study randomization to first extubation (as opposed to successful extubation used for weaning duration) demonstrated a reduction favouring automated systems (mean $\log$ hours $-0.20,-0.34$ to $-0.05, P=0.007 ; 18 \%$ ( $5 \%$ to $29 \%)$ reduction in geometric mean), although statistically significant $(P=0.0005)$ and substantial $\left(I^{2}=68 \%\right)$ heterogeneity was present.

\section{Ventilation duration}

Pooled data from 14 trials reporting total ventilation duration indicated a reduction favouring automated systems (mean log hours $-0.11,-0.18$ to $-0.03, P=0.005$; $10 \%$ (3\% to $16 \%)$ reduction in geometric mean) with no heterogeneity $\left(I^{2}=0 \%, P=0.62\right)$ (Figure 5). There were no subgroup differences according to ICU population, automated system, or the weaning method used in the control arm with broadly overlapping CIs for each subgroup comparison. 


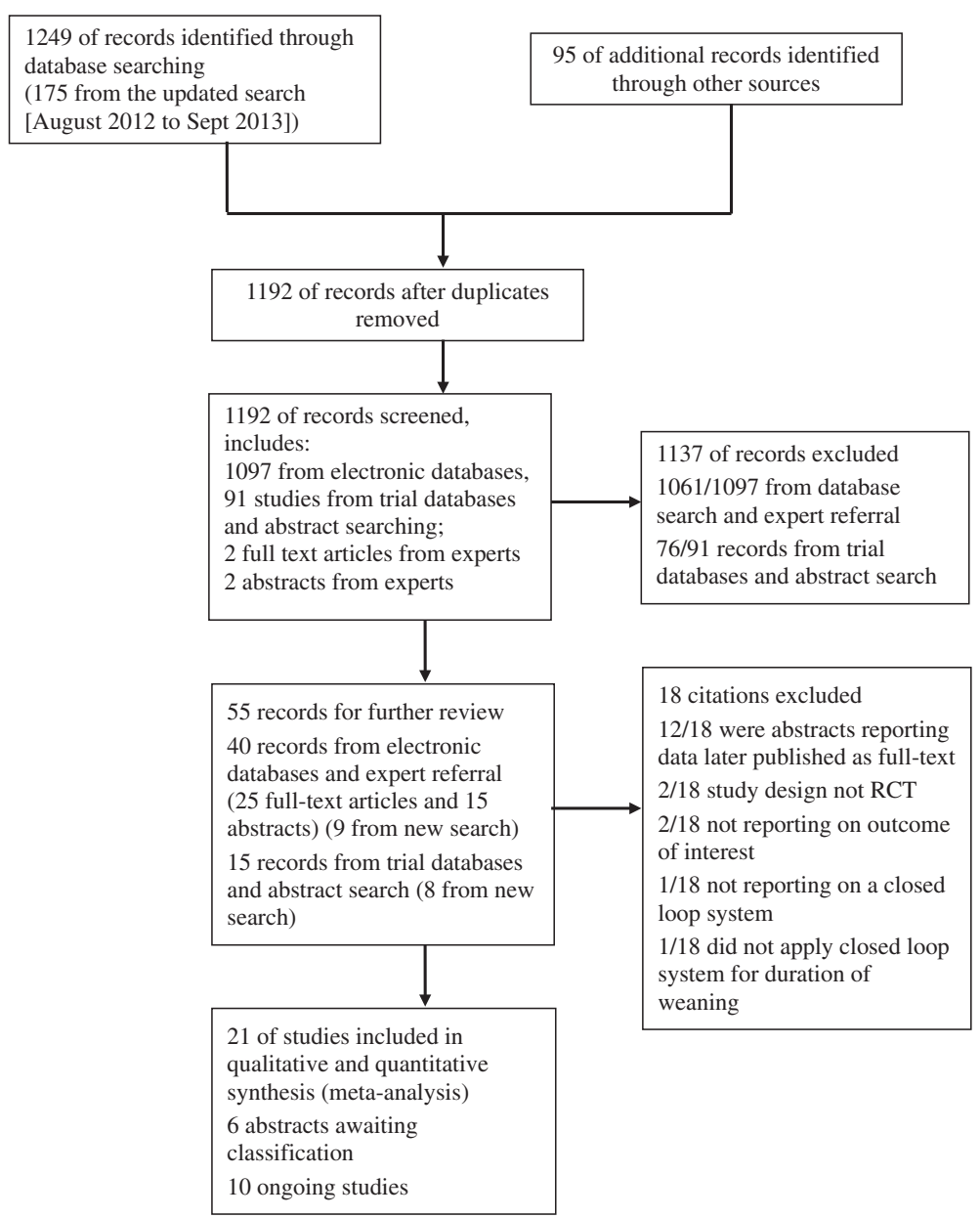

Figure 1 Study flow diagram.

\section{Mortality}

Due to relatively wide CIs (RR $1.04,0.83$ to $1.31, P=0.72$ ), pooled data from 12 trials did not provide strong evidence that automated systems had an effect on mortality when compared to non-automated weaning. Little heterogeneity was noted $\left(I^{2}=3 \%\right)$ (Figure 6$)$.

\section{Duration of hospital and ICU stay}

Pooled data from seven trials reporting hospital LOS found no evidence of effect comparing automated systems and non-automated weaning (mean log days $-0.10,-0.21$ to $0.02, P=0.10 ; 6 \%$ ( $2 \%$ increase to $19 \%$ reduction) reduction in geometric mean) with no heterogeneity $\left(I^{2}=0 \%\right.$, $P=0.52$ ) (Figure 7). Pooled data from 13 trials reporting ICU LOS demonstrated a reduction favouring automated systems (mean log days $-0.08,-0.16$ to $0.00, P=0.05 ; 8 \%$ ( $0 \%$ to $15 \%)$ reduction in geometric mean) with moderate heterogeneity $\left(I^{2}=49 \%, P=0.02\right)$. Pooled data from studies conducted in mixed/medical ICU populations demonstrated reduced ICU stay (mean log days $-0.18,-0.32$ to $-0.04, P=0.01 ; 15 \%$ ( $4 \%$ to $25 \%$ ) reduction in geometric mean) but trials in surgical ICU populations did not (mean log days $0.02,-0.02$ to $0.06, P=0.29 ; 2 \%(2 \%$ reduction to $6 \%$ increase) increase in geometric mean) (Figure 8). Pooled data from Smartcare/PS ${ }^{\mathrm{Tm}}$ trials identified reduced ICU stay (mean log days $-0.26,-0.43$ to -0.09 , $P=0.003 ; 23 \%$ (9\% to $35 \%$ ) reduction in geometric mean) whereas ASV trials did not (mean log days $0.02,-0.02$ to $0.06, P=0.39 ; 2 \%$ ( $2 \%$ reduction to $6 \%$ increase) increase in the geometric mean).

\section{Adverse events}

We did not find strong evidence that automated systems had an effect on reintubation rates in the 13 trials reporting this outcome ( $R R 0.80,0.61$ to $1.05, P=0.1$ ), rates of self-extubation ( $R R 1.24,0.58$ to $2.67, P=0.58$; 9 trials), and non-invasive ventilation after extubation (RR $0.73,0.53$ to $1.02, P=0.07 ; 12$ trials). Prolonged mechanical ventilation (RR $0.51,0.27$ to $0.95, P=0.03 ; 7$ trials) (Figure 9), and tracheostomy (RR 0.67, 0.50 to 0.90, $P=0.008,9$ trials) were reduced in favour of automated systems (Figure 10). 
Table 1 Characteristics of included studies

\begin{tabular}{|c|c|c|c|c|}
\hline Study & Patients & System & Comparator & Weaning success \\
\hline Agarwal, 2013 [29] & 48 adults & ASV & Protocolized: VCV to PSV & Not defined \\
\hline \multirow[t]{2}{*}{ Aghadavoudi, 2012 [20] } & \multirow[t]{2}{*}{81 adults } & \multirow[t]{2}{*}{ ASV } & Protocolized: & \multirow[t]{2}{*}{ Not defined } \\
\hline & & & SIMV (VC) + PS to CPAP & \\
\hline Burns, 2013 [30] & 92 adults & Smartcare/PS & Protocolized: PSV & $\begin{array}{l}\text { Not defined although outcomes reported } \\
\text { for first and successful extubation }\end{array}$ \\
\hline Davis, 1989 [21] & 40 adults & MMV & Protocolized: IMV to CPAP & Not defined \\
\hline Dongelmans, 2009 [19] & 128 adults & ASV & Protocolized: PCV to PSV & Tracheal extubation \\
\hline Hendrix, 2006 [22] & 20 adults & Automode & Protocolized: PRVC to PSV & Not defined \\
\hline Jouvet, 2013 [14] & 30 children & Smartcare/PS & Usual care: PSV & $\begin{array}{l}\text { No further need for ventilation for } 48 \text { hours } \\
\text { after extubation }\end{array}$ \\
\hline \multirow[t]{2}{*}{ Kirakli, 2011 [15] } & \multirow[t]{2}{*}{97 adults } & \multirow[t]{2}{*}{ ASV } & Protocolized: & \multirow{2}{*}{$\begin{array}{l}\text { No further need for ventilation for } 48 \text { hours } \\
\text { after extubation or with a tracheostomy } \\
\text { cannula at day } 28\end{array}$} \\
\hline & & & AC (VC) to PSV & \\
\hline Lellouche, 2006 [23] & 147 adults & Smartcare/PS & Protocolized: PSV or T-piece & $\begin{array}{l}\text { Time from inclusion until successful extubation } \\
\text { (followed by } 72 \text { hours without ventilator support) }\end{array}$ \\
\hline Liu, 2013 [31] & 39 adults & Smartcare/PS & $\begin{array}{l}\text { Protocolized: daily } 30 \mathrm{~min} \text { SBT } \\
\text { of CPAP or PSV }\end{array}$ & 48 hours without reintubation after extubation \\
\hline Petter, 2003 [32] & 34 adults & ASV & Protocolized: SIMV to PSV & Not defined \\
\hline Ramet, 2002 [28] & 18 children & Automode & Protocolized: PRVC to VS & Not defined \\
\hline Rose, 2008 [16] & 102 adults & Smartcare/PS & Usual care: PSV & $\begin{array}{l}\text { No further need for ventilation for } 48 \text { hours } \\
\text { after extubation }\end{array}$ \\
\hline Roth, 2001 [24] & 40 adults & Automode & Protocolized: SIMV to PSV & Not defined \\
\hline Schädler, 2012 [17] & 300 adults & Smartcare/PS & Protocolized: PSV & $\begin{array}{l}\text { Not defined though outcomes reported for } \\
\text { first and successful extubation }\end{array}$ \\
\hline Stahl, 2009 [33] & 60 adults & Smartcare/PS & Usual care: PSV & $\begin{array}{l}\text { Measured reintubation frequency within } \\
48 \text { hours of extubation }\end{array}$ \\
\hline Strickland, 1993 [25] & 17 adults & Non-commercial system & Usual care: SIMV to PSV/CPAP & Not defined \\
\hline Sulzer, 2001 [34] & 36 adults & ASV & Protocolized: SIMV to PSV & Not defined \\
\hline Taniguchi, 2009 [26] & 106 adults & MRV & Protocolized: PCV to PSV & Not defined \\
\hline Walkey [27] & 33 adults & Smartcare/PS & Usual care: A/C to PSV & $\begin{array}{l}\text { No further need for ventilation for } 48 \text { hours } \\
\text { after extubation }\end{array}$ \\
\hline Xirouchaki, 2008 [18] & 208 adults & $\mathrm{PAV}+$ & Protocolized: PSV & Not defined \\
\hline
\end{tabular}

ASV, adaptive support ventilation; CPAP, continuous positive airway pressure; IMV, intermittent mandatory ventilation; MRV, minute respiratory volume; PAV+, proportional assist ventilation; PRVC, pressure regulated volume control; PSV, pressure support ventilation; SBT, spontaneous breathing trial; SIMV, synchronized intermittent mandatory ventilation; VS, volume support.

\section{Sensitivity analyses}

We conducted sensitivity analyses to explore the effects of automated systems prior to log-transforming data. Overall, the pooled weaning duration demonstrated no strong evidence of effect (mean hours -0.75, - 1.85 to $0.34, P=0.18$ ); however, substantial heterogeneity was present $\left(I^{2}=72 \%, P<0.00001\right)$. Subanalyses according to ICU population demonstrated reduced weaning duration in mixed/medical ICU populations (mean hours $-18.75,-32.30$ to $-5.20, P=0.007$ ) but not surgical ICU populations (mean hours $-0.15,-0.70$ to 0.39 , $P=0.58$ ). Weaning duration was reduced in trials of Smartcare/PS ${ }^{\mathrm{mm}}$ (mean hours - 38.46, -58.11 to -18.81 , $P<0.001$ ) but not ASV (mean hours $-0.00,-0.07$ to $0.06, P=0.98$ ) or other automated systems (mean hours $-3.89,-7.71$ to $0.07, P=0.05$ ). Weaning duration was reduced in comparison to usual care (mean hours $-30.49,-60.63$ to $-0.35, P=0.05, I^{2}=52 \%$ ) but not when compared to a protocolized approach. There was no evidence of effect in other measured continuous outcomes. A sensitivity analysis exploring the effect of studies with high risk of bias [27] continued to demonstrate reduced weaning duration using automated systems (mean log hours $-0.37,-0.61$ to $-0.13, P=0.003$; $31 \%$, $12 \%$ to $46 \%$ reduction in geometric mean).

\section{Discussion}

Based on pooled data from 16 eligible trials reporting weaning duration, our study shows automated systems reduced weaning duration by $30 \%$ in the geometric mean. 


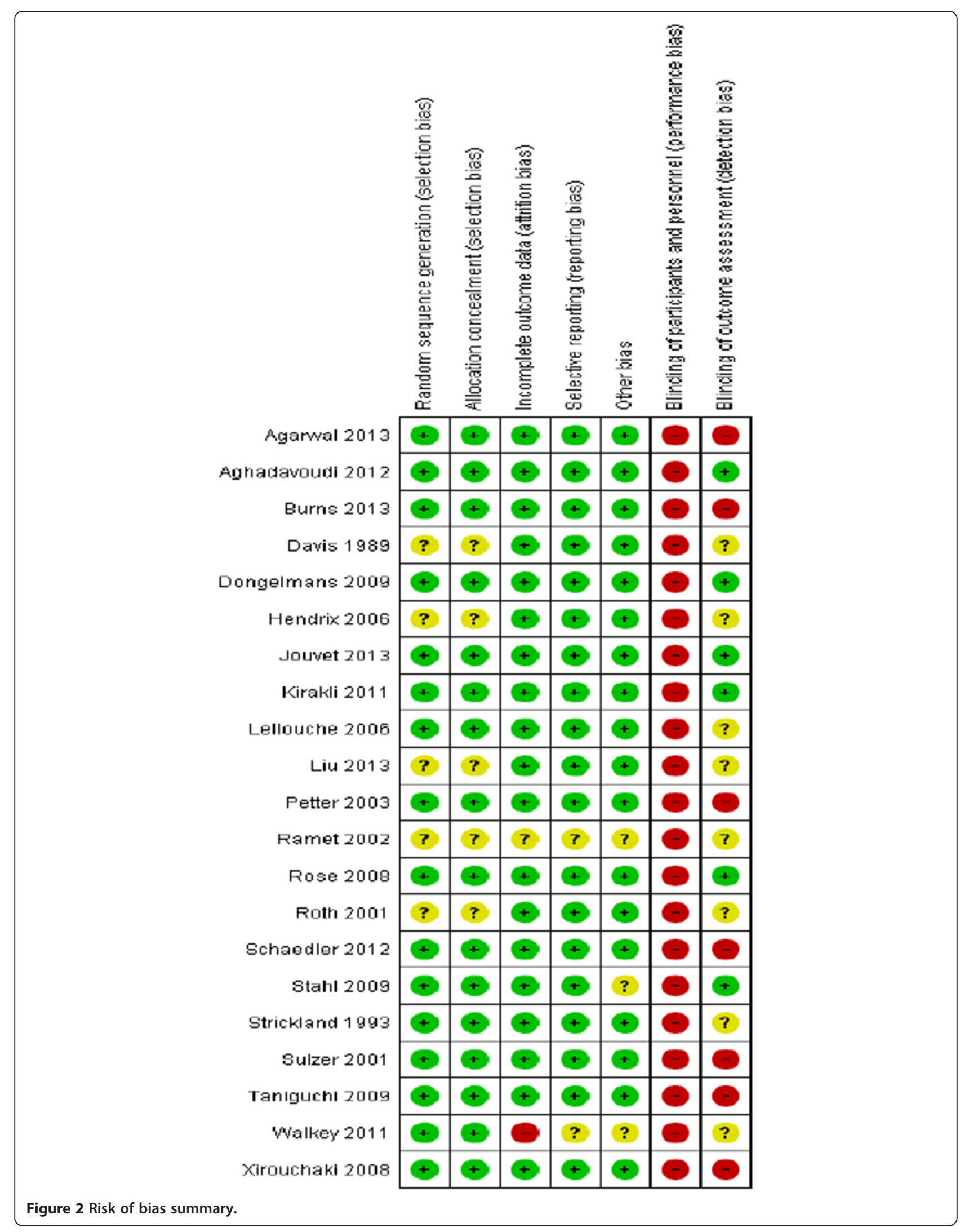




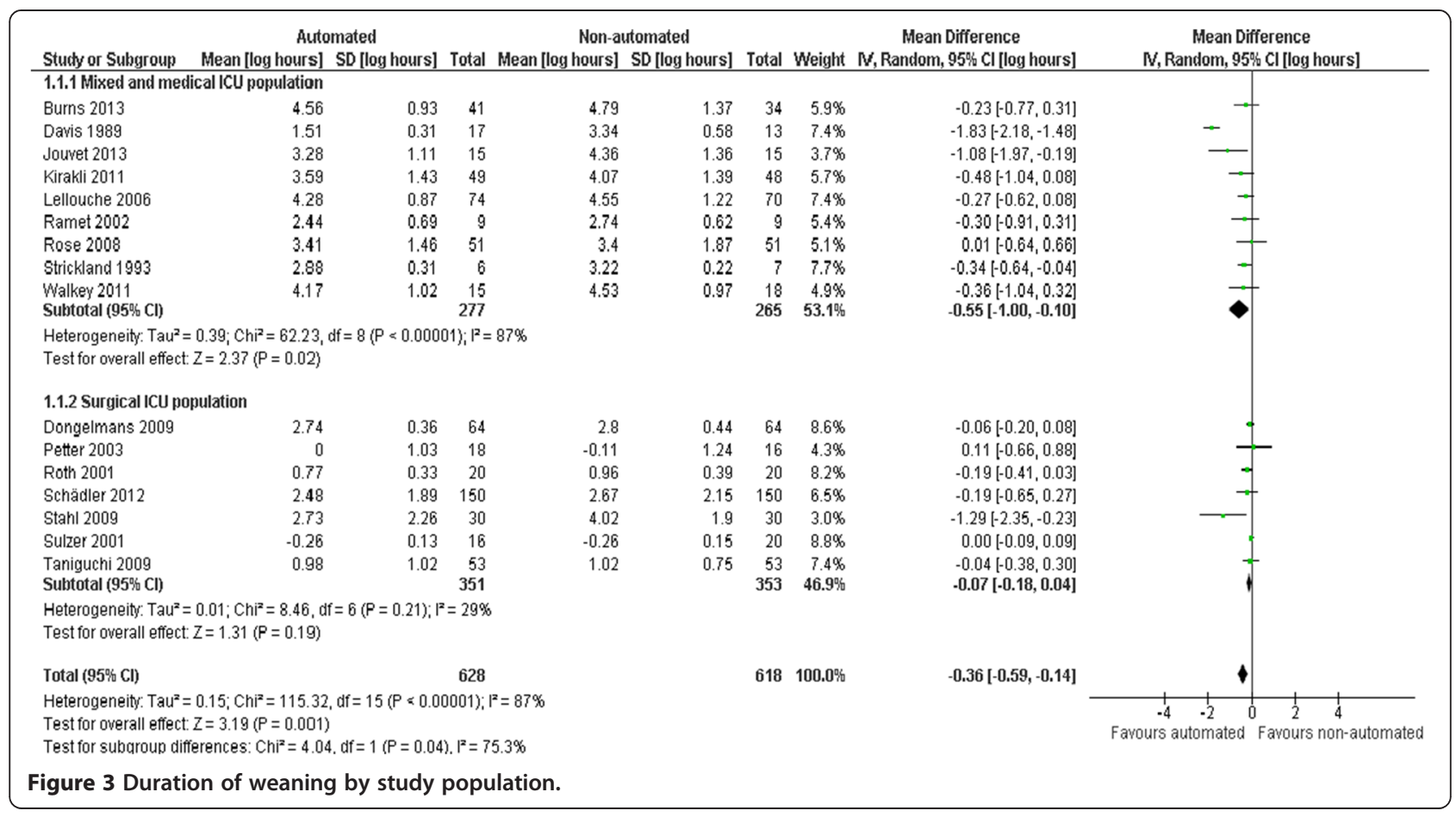

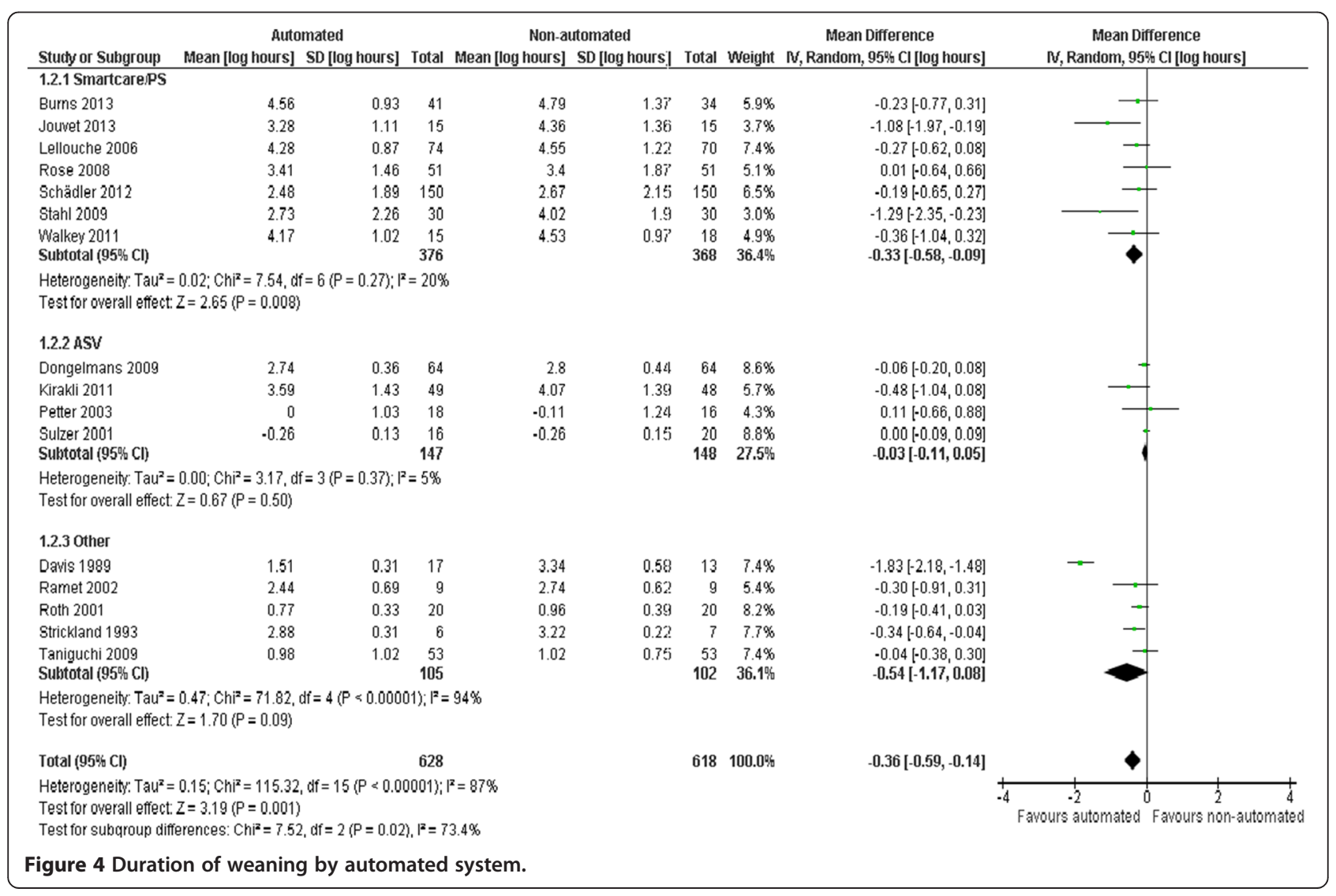




\begin{tabular}{|c|c|c|c|c|c|c|c|c|c|c|}
\hline Study or Subgroup & \multicolumn{3}{|c|}{ Automated } & \multicolumn{3}{|c|}{ Non-automated } & Weight & $\begin{array}{l}\text { Mean Difference } \\
\text { N, Random, 95\% CI [log hours] }\end{array}$ & \multicolumn{2}{|c|}{$\begin{array}{c}\text { Mean Difference } \\
\text { N, Random, } 95 \% \text { Cl [log hours] }\end{array}$} \\
\hline \multicolumn{11}{|c|}{ 1.5.1 Mixed and medical ICU population } \\
\hline Aganwal 2013 & 4.79 & 0.96 & 23 & $4.9 ?$ & 0.89 & 25 & $2.1 \%$ & $-0.18[-0.71,0.35]$ & $\longrightarrow$ & - \\
\hline Burns 2013 & 5.53 & 0.6 & 48 & 5.58 & 1.06 & 43 & $4.4 \%$ & $-0.05[-0.41,0.31]$ & - & - \\
\hline Jouvet 2013 & 5.03 & 0.72 & 15 & 5.35 & 0.9 & 15 & $1.7 \%$ & $-0.32[-0.90,0.26]$ & & - \\
\hline Kirakli 2011 & 4.96 & 0.87 & 49 & 5.08 & 0.87 & 48 & $4.8 \%$ & $-0.12[-0.4 ?, 0.23]$ & — & \\
\hline Lellouche 2006 & 5.06 & 0.75 & 74 & 5.3 & 1.02 & 80 & $6.6 \%$ & $-0.24[-0.53,0.05]$ & - & \\
\hline Liu 2013 & 4.93 & 0.52 & 19 & 5.27 & 0.86 & 20 & $2.9 \%$ & $-0.34[-0.78,0.10]$ & & \\
\hline Ramet 2002 & 4.6 & 0.41 & 9 & 4.36 & 0.71 & 9 & $2.0 \%$ & $0.24[-0.30,0.78]$ & - & \\
\hline Rose 2008 & 4.78 & 0.77 & 51 & 4.83 & 0.86 & 51 & $5.7 \%$ & $-0.05[-0.37,0.27]$ & - & \\
\hline Xirouchaki 2008 & 5.31 & 0.82 & 108 & 5.45 & 0.82 & 100 & $11.5 \%$ & $-0.14[-0.36,0.08]$ & $\rightarrow$ & \\
\hline Subtotal $(95 \% \mathrm{Cl})$ & & & 396 & & & 381 & $41.7 \%$ & $-0.14[-0.25,-0.02]$ & $\bullet$ & \\
\hline \multicolumn{11}{|c|}{$\begin{array}{l}\text { Heterogeneity: } \text { Ta }^{2}=0.00 ; \mathrm{Chi}^{2}=4.11, \mathrm{df}=8(\mathrm{P}=0.85) ; \mathrm{I}^{2}=0 \% \\
\text { Test for overall effect: } Z=2.29(\mathrm{P}=0.02)\end{array}$} \\
\hline \multicolumn{11}{|c|}{ 1.5.2 Surgical ICU population } \\
\hline Aghadavoudi 2012 & 2.05 & 0.36 & 41 & 2.01 & 0.29 & 40 & $28.3 \%$ & $0.04-0.10,0.18]$ & & - \\
\hline Hendrix 2006 & 2.02 & 0.3 & 10 & 2.27 & 0.19 & 10 & $11.8 \%$ & $-0.25[-0.47,-0.03]$ & $\rightarrow$ & \\
\hline Petter 2003 & 0.99 & 0.51 & 16 & 1.16 & 0.29 & 18 & $7.1 \%$ & $-0.1\}[-0.45,0.11]$ & $一$ & \\
\hline Schädler 2012 & 3.79 & 1.24 & 150 & 3.96 & 1.21 & 150 & $7.4 \%$ & $-0.17[-0.45,0.11]$ & & \\
\hline Sulzer 2001 & 1.17 & 0.45 & 16 & 1.4 & 0.76 & 20 & $3.6 \%$ & $-0.23[-0.63,0.17]$ & & \\
\hline Subtotal (95\% Cl) & & & 233 & & & 238 & $58.3 \%$ & $-0.12[-0.26,0.01]$ & - & \\
\hline \multicolumn{11}{|c|}{$\begin{array}{l}\text { Heterogeneity: Tau }{ }^{2}=0.01 ; C h i^{2}=6.34 \text {, df }=4(P=0.18) ; 1^{2}=37 \% \\
\text { Test for overall effiect: } Z=1.74(P=0.08)\end{array}$} \\
\hline Total (95\% Cl) & & & 629 & & & 619 & $100.0 \%$ & $-0.11[-0.18,-0.03]$ & 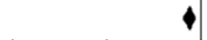 & \\
\hline \multicolumn{6}{|c|}{$\begin{array}{l}\text { Heterogeneity: Tau }{ }^{2}=0.00 ; \mathrm{Chi}^{2}=10.83, \mathrm{df}=13(\mathrm{P}=0.62) ; \mathrm{I}^{2}=0 \% \\
\text { Test for overall effect: } Z=2.81(P=0.005) \\
\text { Test for subaroun differences: } \mathrm{Chi}^{2}=0.03 . \mathrm{df}=1(\mathrm{P}=0.87), \mathrm{I}^{2}=0 \%\end{array}$} & & & & $\begin{array}{lcl}-2 & -1 & 0 \\
& \text { Favours automated }\end{array}$ & Favours non-automated \\
\hline Figure 5 Durati & on of ventilati & ion by auto & omate & ed system. & & & & & & \\
\hline
\end{tabular}

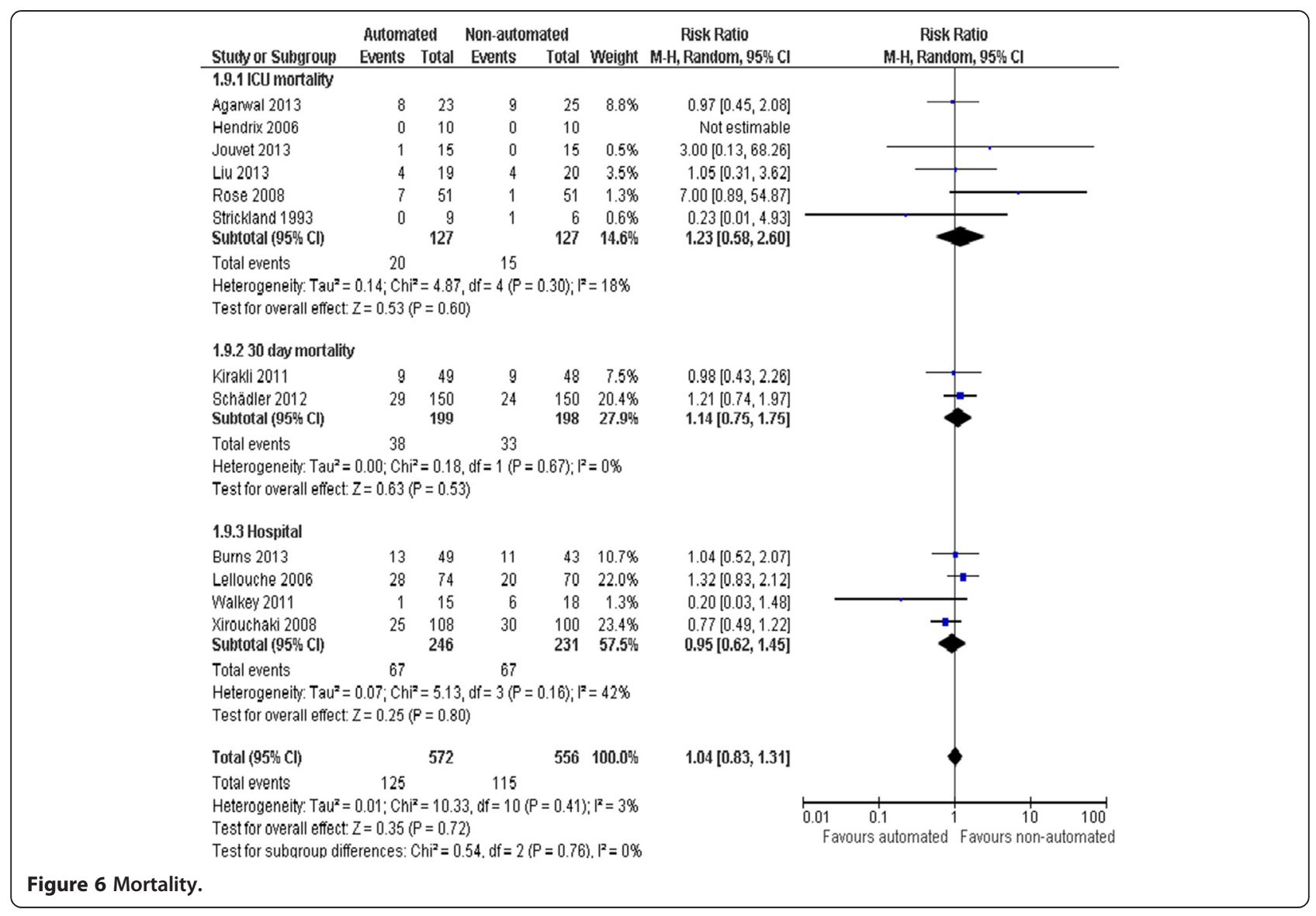




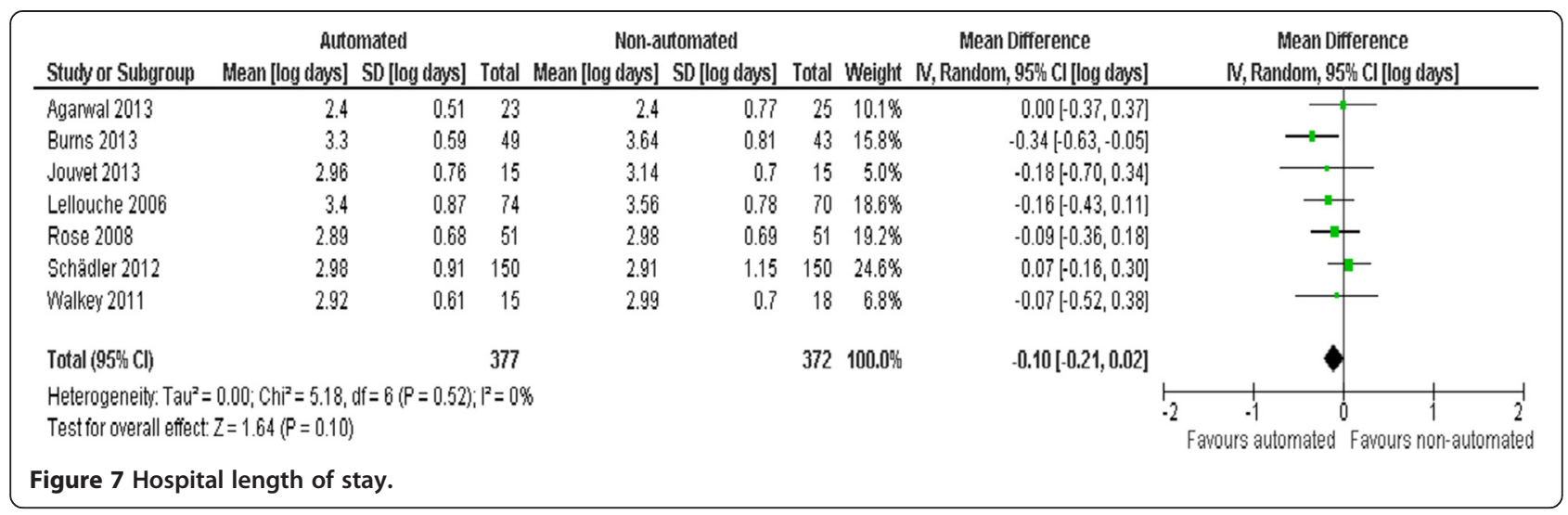

Interpretation of the clinical relevance of a $30 \%$ reduction in the geometric mean is challenging as the number of hours reduced is dependent on the duration of weaning for a given population. As illustration, if the mean duration of weaning was 24 hours as reported in a large international cohort of ventilated patients [39], a 30\% reduction equates to a 7.2 hour reduction in time spent weaning. Subgroup analyses indicated weaning was reduced in trials of mixed/medical ICU populations ( $42 \%$ geometric mean) and Smartcare/PS ${ }^{\mathrm{ma}}$ (28\% geometric mean) but not in surgical ICU populations despite the inclusion of a 300 participant trial that excluded the typical postsurgical 'easy-to-wean' patient. We did not find evidence of effect using automated systems other than Smartcare/PS ${ }^{\mathrm{Tm}}$. The method of weaning in the trial comparator arms (protocol or non-protocolized usual care) did not influence the effect of automated systems on the duration of weaning. Due to substantial heterogeneity caution must be used when interpreting these results. Automated systems also reduced the time to first extubation, ventilation duration, ICU LOS, tracheostomy and prolonged ventilation. There was no strong evidence of an effect on mortality, reintubation, self-extubation, non-invasive ventilation (NIV) postextubation or hospital LOS.

When un-logged data were examined, no difference in weaning duration was detected. However, a statistically significant and clinically meaningful ( $>1$ day) difference in weaning duration was found in trials of mixed and medical ICU populations and SmartCare/PS ${ }^{\mathrm{mm}}$. Disparate findings in primary and sensitivity analyses are due to trials of surgical patients being given more weight in the unlogged analysis because of differences in relative sizes

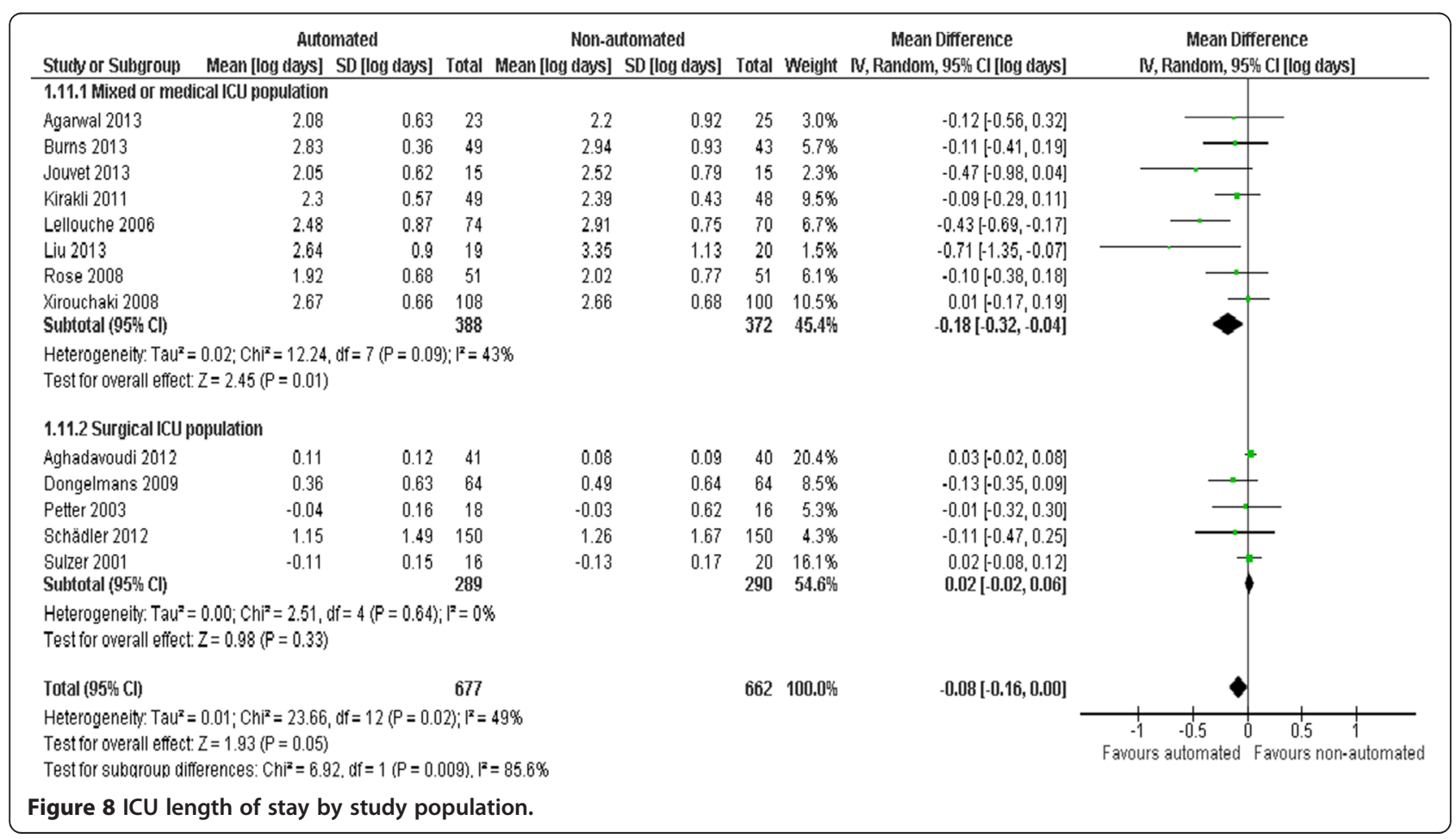




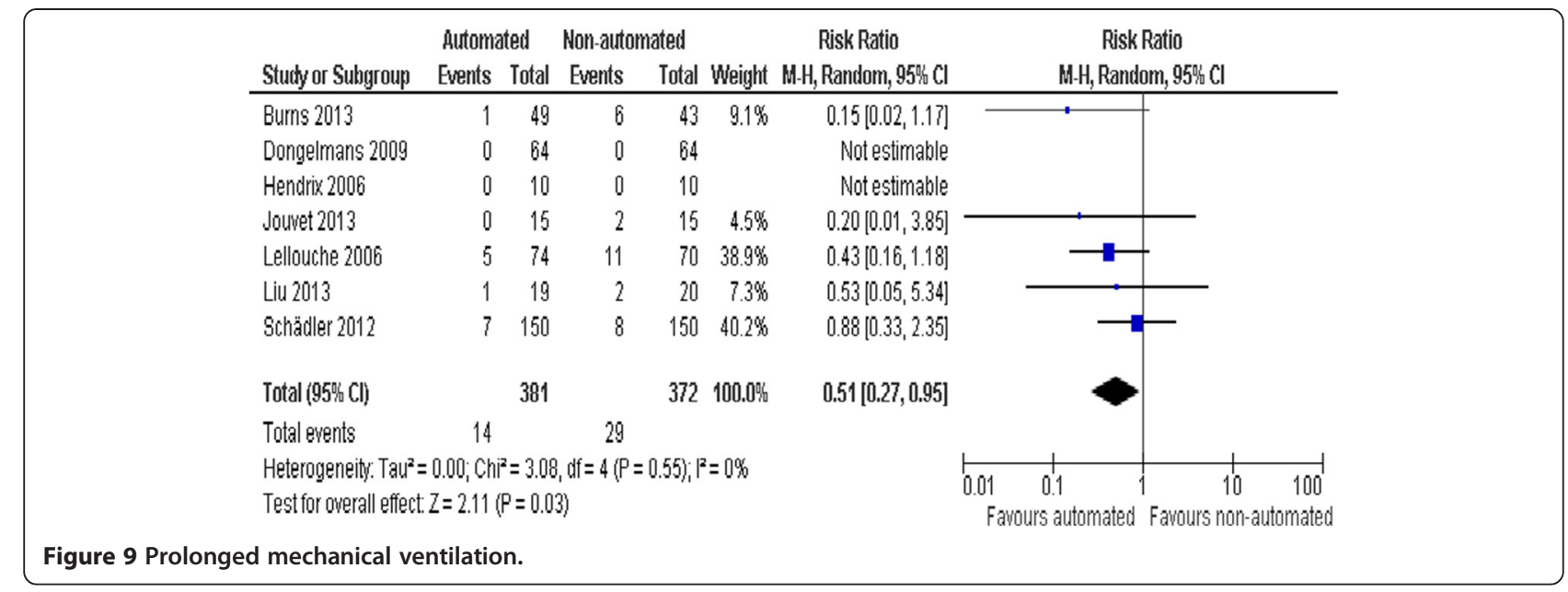

of the standard deviations meaning the overall conclusion is much closer to zero. It is worth noting that pooled estimates from logged and un-logged data in trials of surgical patients are similar and are the same in mixed and medical ICU populations.

Commercial availability of automated systems has led to growing interest and enhanced feasibility in conducting trials such that 13 of the 21 eligible trials were published in the last five years. Most frequently evaluated systems were Smartcare/PS ${ }^{\mathrm{mm}}$ and ASV. While SmartCare/PS ${ }^{\mathrm{mm}}$ has an explicit algorithm for pressure support weaning, ASV does not. Another notable difference in these systems is that ASV automates switching from controlled to spontaneous ventilation if the patient's spontaneous breathing meets the minimum minute ventilation target whereas Smartcare/ $\mathrm{PS}^{\mathrm{Tm}}$ requires clinician recognition of spontaneous breathing and activation of Smartcare/PS ${ }^{\mathrm{mx}}$. This difference is important when considering the potential impact on weaning and ventilation duration. Systems that can switch automatically from controlled to spontaneous breathing may have more influence on overall ventilation duration than those that rely on clinician activation. These systems may also reduce heterogeneity associated with varying entry criteria for weaning commencement. However, we did not detect an effect on ventilation duration in ASV trials due to inclusion of only surgical ICU patients who generally do not experience protracted weaning and ventilation.

We were unable to conduct subgroup analyses according to the weaning classifications arising from the 2005 consensus conference [2] suggesting these are not yet widely adopted as trial inclusion criteria or a priori subgroup analyses. These classifications group patients in terms of number or duration of weaning attempts and may enable better identification of important subgroups or guide patient selection criteria for future trials as opposed to grouping patients according to admission type. We found considerable variation in selection of trial outcomes; indeed, only $71 \%$ reported weaning duration. This suggests the need for consensus on a minimum core outcome set for such trials [40]. We identified only two trials in a pediatric population, one in children

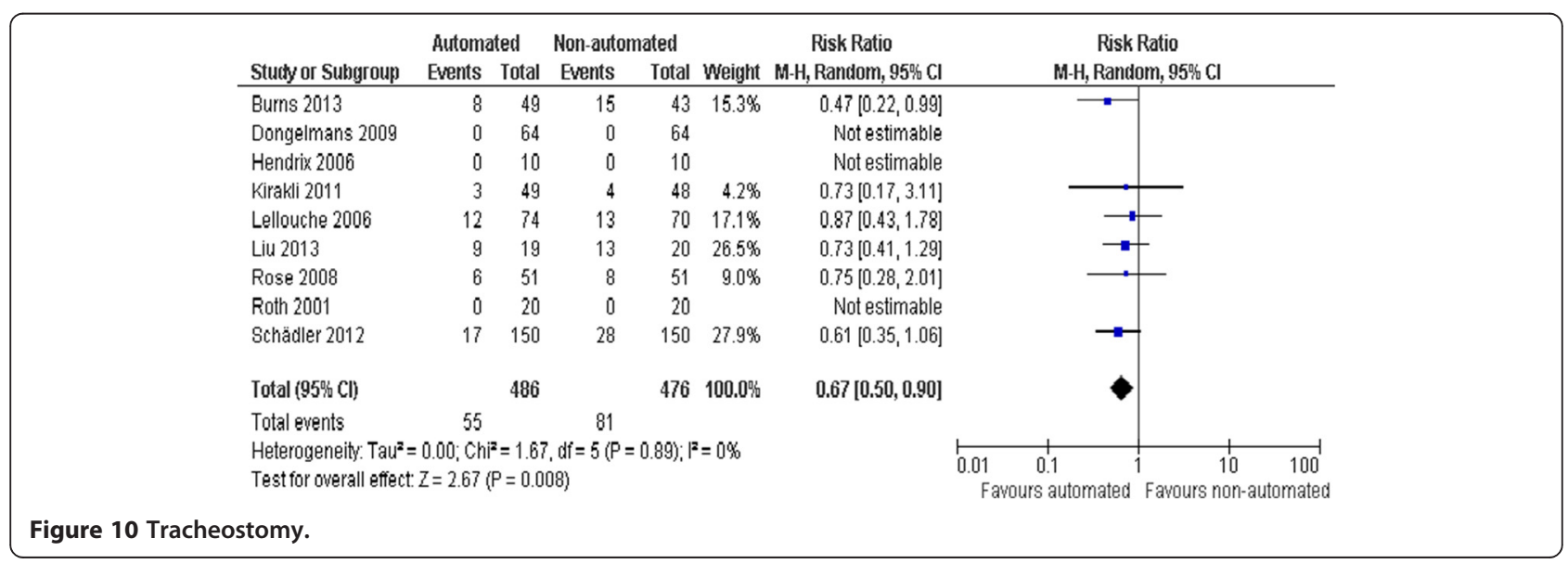


older than two years due to the age and weight limits imposed by SmartCare/PS ${ }^{\mathrm{m}}$. More than $50 \%$ of patients admitted to paediatric ICUs are younger than two years old [41]. This lag in development of automated systems capable of providing appropriate ventilation and weaning to all children probably explains the lack of trials. Given the potential for reduced weaning and ventilation durations, and the small sample size of the two identified trials, there is a need for commercial industry and researchers to further develop and evaluate automated systems adapted to children.

\section{Conclusions}

In conclusion, automated systems may result in clinically meaningful reduced durations of weaning, ventilation and ICU stay. Overall, these systems appear to be safe and can be considered a reasonable approach in the management of ventilator weaning. These potential reductions are more likely to occur in mixed/medical as opposed to surgical ICU populations and with Smartcare $/ \mathrm{PS}^{\mathrm{Tm}}$. Due to limited evidence on automated systems other than Smartcare/PS ${ }^{\mathrm{m}}$ and ASV no conclusions can be drawn regarding their influence on outcomes. The method of weaning to which automated systems was compared (protocolized or non-protocolized usual care) did not influence the effect on weaning duration. Due to substantial heterogeneity in trials reporting weaning duration we believe there is a need for an adequately powered, high quality, multi-centre trial in an adult patient population that excludes patients classified as 'simple to wean' with detailed description of ICU organizational characteristics and weaning/sedation strategies in the comparator arm as these may be contributing to clinical heterogeneity. As little data exists on other automated systems and ASV in prolonged weaning, we are cautious to recommend which should be selected for investigation.

\section{Key messages}

- Automated systems may reduce weaning and ventilation duration particularly for mixed/medical ICU populations.

- These systems may also reduce the need for tracheostomy and prolonged mechanical ventilation.

- Thirteen of the 21 eligible trials were published in the last five years indicating growing interest in automated closed loop systems.

- There is a need for commercial industry and researchers to further develop and evaluate automated systems adapted to children.

\section{Additional file}

Additional file 1: Description of automated systems.

\section{Abbreviations}

ASV: adaptive support ventilation; Cl: confidence interval; ICU: intensive care unit; IQR: interquartile range; LOS: length of stay; PAV: proportional assist ventilation; RR: risk ratio; SD: standard deviation.

\section{Competing interests}

Bronagh Blackwood, Chris R Cardwell and Danny F McAuley declare that they have no competing interests. Louise Rose has completed an RCT comparing automated weaning using SmartCare/PS ${ }^{\mathrm{TM}}$ with usual care that was included in this systematic review. Dr Rose received no funding from Draeger Medical for this study. SmartCare/PS software, and associated technical upgrades for two ventilators, were provided free of charge to the Intensive Care Unit of The Royal Melbourne Hospital by Draeger Medical, Australia. Marcus J Schultz received a research fund of 12,000 Euro for a study on weaning with ASV by Hamilton Medical, Switzerland. Hamilton Medical provided Prof Schultz's hospital research group with 12,000 Euro for performing a study of weaning with ASV in cardiac surgical patients. The money was used to buy equipment, a computer, statistician time, and to cover costs involved with the presentation of the results at a scientific meeting and publication of an article. Philippe Jouvet has completed an RCT comparing automated weaning using SmartCare/PS ${ }^{\text {TM }}$ with usual care, included in this systematic review. Dr Jouvet did not receive any funding from Draeger for this study, although, for the purposes of the study, Draeger Medical provided one ventilator. Dr Jouvet received two grants as support from Hamilton Medical for two clinical studies, including a prospective trial on automation of weaning with ASV in children (NCT01095406) and a clinical study on criteria for mechanical ventilation adjustments in children. These two studies are not eligible for inclusion in the systematic review. Dr Jouvet also conducted a clinical trial on weaning with NAVA in infants (NCT00603174) that is not eligible for inclusion in the systematic review. Dr Jouvet did not receive any funding from Maquet for this study, although, for the purposes of the study, Maquet provided one ventilator.

\section{Authors' contributions}

LR conceived of the review, screened search results and undertook manual searches and entered data in to Revman. MS appraised data quality and abstracted data. CC assisted with statistical analyses. PJ screened search results and undertook manual searches. DM arbitrated decisions pertaining to study inclusion and data abstraction. BB conceived of the review, appraised data quality and abstracted data. All authors assisted with writing the review protocol, interpretation of data and manuscript. All authors have contributed to protocol design, data collection, data analysis and report writing. All authors read and approved the final manuscript.

\section{Acknowledgements}

This study was funded by a Knowledge Synthesis grant from the Canadian Institutes of Health Research. The funder has had no role in the design, in the collection, analysis, and interpretation of data; in the writing of the manuscript; and in the decision to submit the manuscript for publication. We would like to acknowledge the support of the Cochrane Anaesthesia Review Group for their assistance, support and editorial advice. This article is published in the Cochrane Database of Systematic Reviews (CDSR) 2014, Issue 6, CD009325 (see www.thecochranelibrary.com for information). Cochrane Reviews are regularly updated as new evidence emerges and in response to feedback, and the CDSR should be consulted for the most recent version of the review.

\section{Author details}

'Department of Critical Care Medicine, Sunnybrook Health Sciences Centre, 2075 Bayview Ave, Toronto, ON M4N 3M5, Canada. 'Lawrence S. Bloomberg Faculty of Nursing, University of Toronto, 155 College St, Toronto, Ontario M5T IP8, Canada. ${ }^{3}$ Provincial Centre of Weaning Excellence, Toronto East General Hospital, Toronto, Canada. ${ }^{4}$ Li Ka Shing Institute, St Michael's Hospital, 30 Bond St, Toronto, ON M5B 1W8, Canada. ${ }^{5}$ West Park Healthcare Centre, University of Toronto, 155 College St, Toronto, Ontario M5T IP8, Canada. ' ${ }^{6}$ aboratory of Experimental Intensive Care and Anesthesiology, Academic Medical Center, University of Amsterdam, Meibergdreef 9, 1105 AZ Amsterdam, Netherlands. ${ }^{7}$ Centre for Public Health, Queen's University Belfast, University Rd, Belfast BT7 1NN, UK. ${ }^{8}$ Department of Pediatrics, Sainte-Justine Hospital, University of Montreal, 3175 Chemin de la Côte-Sainte-Catherine, Montreal, QC H3T 1C5, Canada. ${ }^{9}$ Regional Intensive 
Care Unit, Royal Victoria Hospital, Centre for Infection and Immunity, Queen's University of Belfast, University Rd, Belfast BT7 $1 \mathrm{NN}$, UK. ${ }^{10}$ Centre for Infection and Immunity, School of Medicine, Dentistry \& Biomedical Sciences, Queen's University Belfast, University Rd, Belfast BT7 1NN, UK.

\section{Received: 9 May 2014 Accepted: 19 January 2015 Published online: 24 February 2015}

\section{References}

1. Maclntyre NR. Evidence-based guidelines for weaning and discontinuing ventilatory support. Chest. 2001;120:375S-95S.

2. Boles JM, Bion J, Connors A, Herridge M, Marsh B, Melot C, et al. Weaning from mechanical ventilation. Eur Resp J. 2007;29:1033-56.

3. Ely EW, Meade MO, Haponik EF, Kollef MH, Cook DJ, Guyatt GH, et al. Mechanical ventilator weaning protocols driven by nonphysician health-care professionals: evidence-based clinical practice guidelines. Chest. 2001;120:454S-63S.

4. Blackwood B, Alderdice F, Burns K, Cardwell C, Lavery G, O'Halloran P. Protocolized versus non-protocolized weaning for reducing the duration of mechanical ventilation in critically ill adult patients. Cochrane Database Syst Rev. 2010;5:CD006904.

5. Lellouche F, Brochard L. Advanced closed loops during mechanical ventilation (PAV, NAVA, ASV, SmartCare). Best Pract Res Clin Anaesthesiol. 2009;23:81-93.

6. Zilberberg M, de Wit M, Shorr A. Accuracy of previous estimates for adult prolonged acute mechanical ventilation volume in 2020: update using 2000-2008 data. Crit Care Med. 2012;40:18-20.

7. Wunsch H, Linde-Zwirble W, Angus D, Hartman M, Milbrandt E, Kahn J. The epidemiology of mechanical ventilation use in the United States. Crit Care Med. 2010:38:1947-53.

8. Zolnierek C, Steckel C. Negotiating safety when staffing falls short. Crit Care Nurs Clin North Am. 2010;22:261-9.

9. Angus D, Shorr A, White A, Deremsiziv T, Schmitz R, Kelley M. Critical care delivery in the United States: distribution of services and compliance with leapfrog recommendations. Crit Care Med. 2006;34:1016-24.

10. Rose L, Schultz M, Cardwel C, Jouvet P, McAuley D, Blackwood B. Automated versus non-automated weaning for reducing the duration of mechanical ventilation for critically ill adults and children (review). Cochrane Database Syst Rev. 2014;6:CD009235.

11. Leclerc F, Noizet O, Botte A, Binoche A, Chaari W, Sadik A, et al. Weaning from invasive mechanical ventilation in pediatric patients (excluding premature neonates). Arch Pediatrics. 2010;17:399-406.

12. Cochrane Handbook for Systematic Reviews of Interventions 5.1.0. In: Higgins J, Green S: (eds) The Cochrane Collaboration. www.cochranehandbook.org. 2011, Section 7.7.3.5.

13. DeMets D. Methods for combining randomized clinical trials. Stat Med. 1987;6:341-50

14. Jouvet P, Payen $\mathrm{V}$, Gauvin F, Emeriaud G, Lacroix J. Weaning children from mechanical ventilation with a computer-driven protocol: a pilot trial. Intensive Care Med. 2013;39:919-25.

15. Kirakli C, Ozdemir I, Ucar Z, Cimen P, Kepil S, Ozkan S. Adaptive support ventilation for faster weaning in COPD: a randomised controlled trial. Eur Respir J. 2011:38:774-80.

16. Rose L, Presneill J, Johnston L, Cade J. A randomised, controlled trial of conventional versus automated weaning from mechanical ventilation using SmartCare/PS. Intensive Care Med. 2008:34:1788-95.

17. Schädler D, Engel C, Elke G, Pulletz S, Haake N, Frerichs I, et al. Automatic control of pressure support for ventilator weaning in surgical intensive care patients. Am J Respir Crit Care Med. 2012;185:637-44.

18. Xirouchaki N, Kondili E, Vaporidi K, Xirouchakis G, Klimathianaki M, Gavriilidis G, et al. Proportional assist ventilation with load-adjustable gain factors in critically ill patients: comparison with pressure support. Intensive Care Med. 2008:34:2026-34

19. Dongelmans D, Veelo D, Paulus F, de Mol B, Korevaar J, Kudoga A, et al. Weaning automation with adaptive support ventilation: a randomized controlled trial in cardiothoracic surgery patients. Anesth Analg. 2009;108:565-71.

20. Aghadavoudi $\mathrm{O}$, Kamran M, Masoudifar M. Comparison of two modes of ventilation after fast-track cardiac surgery: adaptive support ventilation versus synchronized intermittent mandatory ventilation. Pakistan J Med Sci. 2012;28:303-8
21. Davis $S$, Potgieter $P$, Linton D. Mandatory minute volume weaning in patients with pulmonary pathology. Anaesthesia Intensive Care. 1989;17:170-4.

22. Hendrix H, Kaiser M, Yusen R, Merk J. A randomized trial of automated versus conventional protocol-driven weaning from mechanical ventilation following coronary artery bypass surgery. Eur J Cardiothor Surg. 2006;29:957-63.

23. Lellouche F, Mancebo J, Jolliet P, Roeseler J, Schortgen F, Dojat M, et al. A multicenter randomized trial of computer-driven protocolized weaning from mechanical ventilation. Am J Respir Crit Care Med. 2006;174:894-900.

24. Roth H, Luecke T, Lansche G, Bender H, Quintel M. Effects of patient-triggered automatic switching between mandatory and supported ventilation in the postoperative weaning patients. Intensive Care Med. 2001;27:47-51.

25. Strickland Jr JH, Hasson JH. A computer-controlled ventilator weaning system. A clinical trial. Chest. 1993;103:1220-6.

26. Taniguchi C, Eid R, Saghabi C, Souza R, Silva E, Knobel E, et al. Automatic versus manual pressure support reduction in the weaning of post-operative patients: a randomised controlled trial. Crit Care. 2009;13:R6.

27. Walkey A, Reardon C. Clinical trial of a computer driven weaning system for patients requiring mechanical ventilation. Available from https://clinicaltrials. gov/ct2/show/NCT00606554intr=computer+driven+weaning\&rank=1.

28. Ramet J, Van Herreweghe I, Idrissi S, Huyghens L. Computerized switch of ventilation mode during weaning [abstract]. Intensive Care Med. 2002;28:S123.

29. Agarwal S, Srinivasan A, Aggarwal A, Gupta D. Adaptive support ventilation for complete ventilatory support in acute respiratory distress syndrome: a pilot, randomized controlled trial. Respirology. 2013;18:1108-15.

30. Burns KE, Meade MO, Lessard MR, Hand L, Zhou Q, Keenan SP, et al. Wean earlier and automatically with new technology (the WEAN study). A multicenter, pilot randomized controlled trial. Am J Respir Crit Care Med. 2013;187:1203-11.

31. Liu L, Xu X, Yang Y, Huang Y, Liu S, Qiu H. Computer-driven automated weaning reduces weaning duration in difficult-to-wean patients. Chin Med J. 2013;126:1814-8.

32. Petter A, Chiolero R, Cassina T, Chassot PG, Muller XM, Revelly JP. Automatic respirator weaning with adaptive support ventilation: the effect on duration of endotracheal intubation and patient management. Anesth Analgesia. 2003:97:1743-50

33. Stahl C, Dahmen G, Ziegler A, Muhl E. Protocolized automated versus non-protocolized physician-directed weaning from mechanical ventilation: a controlled clinical trial. Intensivemed Prax. 2009;46:441-446.

34. Sulzer CF, Chiolero R, Chassot PG, Mueller XM, Revelly JP. Adaptive support ventilation for fast tracheal extubation after cardiac surgery: a randomized controlled study. Anesthesiology. 2001;95:1339-45.

35. Hozo S, Djulbegovic B, Hozo I. Estimating the mean and variance from the median, range, and the size of a sample. BMC Med Res Methodol. 2005;5:13.

36. Bland J, Altman D. The use of transformation when comparing two means. BMJ. 1996:312:1153.

37. The Nordic Cochrane Centre, The Cochrane Collaboration. Review Manager (RevMan), Version 5.2 for Windows (computer program). 2012, Copenhagen.

38. Egger M, Davey Smith G, Schneider M, Minder C. Bias in meta-analysis detected by a simple, graphical test. BMJ. 1997;315:629-34.

39. Esteban A, Ferguson N, Meade M, Frutos-Vivar F, Apezteguia C, Brochard L, et al. Evolution of mechanical ventilation in response to clinical research. Am J Respir Crit Care Med. 2008;177:170-7.

40. Clarke M. Standardising outcomes for clinical trials and systematic reviews. Trials. 2007:8:39.

41. Payen $V$, Jouvet P, Lacroix J, Ducruet T, Gauvin F. Risk factors associated with increased length of mechanical ventilation in children. Ped Crit Care Med. 2012;13:152-7. 